\title{
Effects of Remote Substituents on Electrochemical Reduction of
}

\section{$\mathrm{X}-\mathrm{PhCH}=\mathrm{NPhCH}=\mathrm{CHPh}-\mathrm{Y}$}

\author{
Zhengjun Fang ${ }^{1,2}$, Ru Zhang ${ }^{1,2}$, Wanmin Liu ${ }^{1,2}$, Qiang Tao ${ }^{1,2}$, \\ Bianlin Zhang ${ }^{l}$ and Donghong $\mathrm{Yu}^{2,3, *}$ \\ ${ }^{1)}$ Hunan Provincial Key Laboratory of Environmental Catalysis \& Waste Recycling, School \\ of Chemistry and Chemical Engineering, Hunan Institute of Engineering, Xiangtan \\ 411104, China \\ ${ }^{2)}$ Department of Chemistry and Bioscience, Aalborg University, Aalborg 9220, Denmark \\ ${ }^{3)}$ Sino-Danish Center for Education and Research (SDC), Aarhus, 8000, Denmark \\ *)E-mail:yu@bio.aau.dk
}

Schiff-bases have prospective applications in functional technologies such as organic photovoltaic [1] and other molecular electronic devices [2]. Therefore, for the design of molecular optoelectronic devices of high efficiency, it is hence of great interest and significance to understand the relationship between structure and electrochemical property of Schiff bases in the molecular level [3]. However, Schiff base displays a high degree of complexity that makes it difficult and expensive to elaborate this in a large scale. Consequently, quantitative structure-property relationship (QSPR) focusing on simple, costeffective and easily scalable Schiff bases has emerged [4].

In this paper substituent effects on the electrochemical properties of $\mathrm{X}$ $\mathrm{PhCH}=\mathrm{NPhCH}=\mathrm{CHPh}-\mathrm{Ycompound}$ with long conjugated backbone were studied from the viewpoint of reduction potentials $\left(\mathrm{E}_{(\mathrm{red})}\right)$. The reduction potentials of 28 compounds of this kind were determined by cyclic voltammetry, and the effects of $\mathrm{X}$ and $\mathrm{Y}$ as substituents on $\mathrm{E}_{(\mathrm{red})}$ were explored separately by experimental and computational methods, and their contribution on $\mathrm{E}_{(\mathrm{red})}$ was investigated. The results show that the introduction of a styryl group promotes conjugation, leading to a shift of $\mathrm{E}_{(\mathrm{red})}$ in the positive direction. Electronic effects, especially resonance effects of substituents, play a key role in tuning the $\mathrm{E}_{(\mathrm{red})}$ of XBASBY. Also, with the increase of conjugated chain length, $\mathrm{E}_{(\mathrm{red})}$ becomes more dependent on the effects of the excited states of $\mathrm{X}$ and $\mathrm{Y}$. A quantitative model is developed to express the effects of remote substituents on $\mathrm{E}_{(\mathrm{red})}$, which is confirmed to be reliable by lee-one-out method. In particular, the remote substituents $Y$ cause effects on $E_{(\text {red })}$ enhancement rather than attenuation. The experimental results can be clarified based on the calculation results of frontier molecular orbital and band gap energies. Also, the relative energies of radical anion were proposed by DFT method to explain the role of the styryl group (Figure 1). 


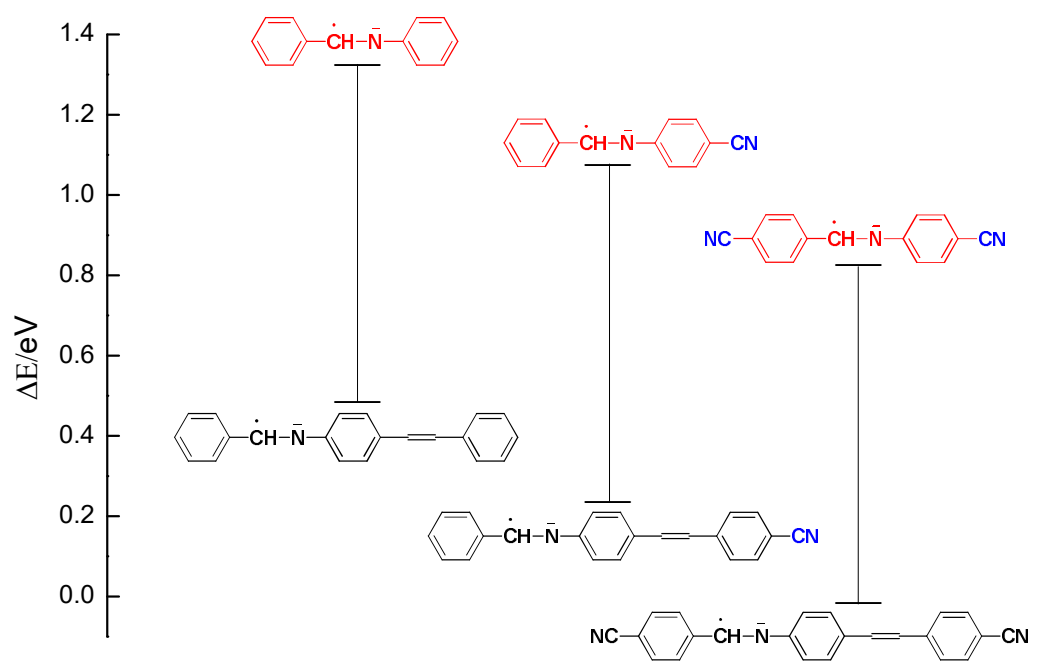

Figure1. Radical anion of XBAY and XBASBY and their energies relative to CNBASBCN, whose energy level is defined as zero.

Given the effects of styryl group and substituents, it is anticipated that the $\mathrm{E}_{\text {(red) }}$ of Schiff bases can be adjusted by controlling the distance between substituents and $-\mathrm{CH}=\mathrm{N}$ - center. It is envisaged that by extending the molecular conjugated length, the electrochemical properties of Schiff-base compounds can be effectively tuned for applications in areas of organic photovoltaic-like molecular electronic devices.

\section{References.}

[1] A. Faurie, J. Grolleau, F. Gohier, M. Allain, S. Legoupy, P. Frere, Eur. J. Org. Chem19. 2707-2714 (2017).

[2] M. Zambianchi, L. Favaretto, M. Durso, C. Bettini, A. Zanelli, I. Manet, M. Gazzano, L. Maini, D. Gentili, S. Toffanin, J. Mater. Chem. C3, 121-131(2015).

[3] J.X. Liao, H.B. Zhao, Z.D. Cai, Y.J. Xu, F.G.F. Qin, Q. Zong, F. Peng, Y.T. Fang.. Org. Electron61, 215-222(2018).

[4] A. Cidlina, V. Novakova, M. Miletin, P. Zimcik. Dalton. Trans44, 6961-6971(2015). 